\title{
Quality of bull spermatozoa after preparation by single-layer centrifugation
}

\author{
Lavanya Goodla, ${ }^{\star} †$ Jane M. Morrell, ${ }^{\star 1}$ Yulnawati Yusnizar, $¥ \S$ Hans Stålhammar,\# and Anders Johannisson† \\ *Department of Clinical Sciences, Division of Reproduction, \\ †Department of Anatomy, Physiology, and Biochemistry, and \\ ¥Department of Animal Breeding and Genetics, Swedish University of Agricultural Sciences (SLU), Box 7054, 75007 Uppsala, Sweden \\ $\S$ Research Center for Biotechnology, Indonesian Institute of Sciences, Jl. Raya Bogor Km. 46, Cibinong 16911, Indonesia \\ \#Viking Genetics, Box 64, SE-532 21, Skara, Sweden
}

\section{ABSTRACT}

The present study aimed to evaluate the effect of single-layer centrifugation (SLC) through a species-specific colloid (Androcoll-B; patent pending, J. M. Morrell) on bull sperm quality. Computer-assisted sperm analysis of motility and flow cytometric analysis of sperm viability (SYBR-14/propidium iodide staining), chromatin integrity (acridine orange staining), reactive oxygen species production [Hoechst 33258-hydroethidine-2', $7^{\prime}$ dichlorodihydrofluorescein diacetate (HO-HE-DCFDA) staining], mitochondrial membrane potential (staining with JC-1 probe), and protein tyrosine phosphorylation (specific antibody staining) were performed on unselected and SLC-selected sperm samples. Single-layer centrifugation of bull spermatozoa resulted in the selection of a sperm population that had high mitochondrial membrane potential, a higher content of phosphorylated protein, and more reactive oxygen species than control samples. Sperm chromatin damage was lower in the SLC samples although sperm viability and motility did not differ between SLC samples and controls. These observations suggest that SLC of bull semen in a soybean-containing extender improved some, but not all, parameters of sperm quality.

Key words: bull spermatozoa, flow cytometry, mitochondrial membrane potential, protein tyrosine phosphorylation, single-layer centrifugation

\section{INTRODUCTION}

Artificial insemination is the most widely used assisted reproduction technique for breeding dairy cattle, with fertilization rates ranging from 80 to $95 \%$. However, calving rates in Sweden are usually between 50 and $60 \%$ and declining year by year (Morrell and Rodriguez-Martinez, 2011). Artificial insemination can increase pregnancy rate or the efficiency of assisted reproduction only if the insemination dose contains suf-

Received October 15, 2013.

Accepted December 21, 2013.

${ }^{1}$ Corresponding author: Jane.Morrell@slu.se ficient numbers of viable spermatozoa that are capable of reaching the site of fertilization.

Fertility is a multifaceted phenomenon, involving both male and female factors. Traditionally, the fertility of breeding sires has been attributed to sperm quality, whereas the contribution of the other major component of semen, seminal plasma, has largely been ignored.

Selecting the most robust spermatozoa from the rest of the ejaculate could be an approach to improve sperm quality. A new sperm selection technique has been developed that selects good quality spermatozoa and removes them from the seminal plasma (Morrell and Rodriguez-Martinez, 2009). This technique, singlelayer centrifugation (SLC) through species-specific colloid formulations (Androcoll; patent pending, J. M. Morrell), resulted in stallion and Blanc-Celtibérica buck sperm samples with better motility and normal morphology and sperm chromatin integrity compared with the corresponding unselected samples (Morrell et al., 2009a,b; Jiménez-Rabadán et al., 2012).

The growing availability of fluorochromes and compounds conjugated to fluorescent probes has stimulated interest in a variety of flow cytometric methods for addressing many properties of sperm, including viability, reactive oxygen species (ROS) production, mitochondrial membrane integrity, and chromatin structure (Hossain et al., 2011). Development of computer-assisted sperm analysis (CASA) systems has provided a means to obtain large amounts of objective information of the kinematics and morphometric characteristics of a semen sample (Verstegen et al., 2002).

Previous research with bull spermatozoa showed that the proportion of spermatozoa with normal morphology in the ejaculates relates to fertility (Söderquist et al., 1996, 1997) and that this proportion varies not only between males, but also with age and breed (Hallap et al., 2005, 2006). Therefore, if the same relationships between sperm quality of the original semen sample and yield after SLC are seen for bulls as for stallions (Morrell et al., 2014), processing bull samples by SLC could help to improve pregnancy rates following AI. 
With this background, the present study was designed to evaluate the changes in sperm quality produced by SLC, in terms of morphology, plasma membrane integrity, motility, chromatin structure, ROS production, mitochondrial membrane integrity, and protein tyrosine phosphorylation in bull semen samples.

\section{MATERIALS AND METHODS}

\section{Semen Samples}

Semen was collected once weekly from 20 bulls at a commercial bull stud (Viking Genetics, Falkenberg, Sweden) according to standard husbandry practices. The semen was extended in warm $\left(35^{\circ} \mathrm{C}\right)$ Andromed (Minitüb GmbH, Tiefenbach, Germany) to provide a sperm concentration of $69 \times 10^{6}$ spermatozoa/mL, according to the company's routine practice for preparing semen for freezing. A $25-\mathrm{mL}$ aliquot of this extended semen in a universal tube (Sarstedt AB, Helsingborg, Sweden) was placed in a Styrofoam box with a cold pack and transported overnight to the laboratory at the Swedish University of Agricultural Sciences (Uppsala, Sweden). The time between semen collection and subsequent SLC and analysis was approximately 25 to $28 \mathrm{~h}$. Samples from 3 ejaculates per bull were available.

\section{Single-Layer Centrifugation}

The SLC method was performed according to the protocol previously described for stallion semen (Morrell et al., 2009a), with slight modifications; that is, the colloid formulation was specifically designed for bull spermatozoa (Androcoll-B; patent pending, J. M. Morrell). The sperm concentration in the semen samples was adjusted to $50 \times 10^{6} / \mathrm{mL}$ for SLC, with $15 \mathrm{~mL}$ of sample being layered on top of $15 \mathrm{~mL}$ of Androcoll-B in a centrifuge tube. The preparation was centrifuged twice at $300 \times g$ for $20 \mathrm{~min}$ each at room temperature (approximately $23^{\circ} \mathrm{C}$ ), after which the supernatant and most of the colloid was discarded and the sperm pellet was transferred to a clean tube containing Andromed extender. Aliquots of both unselected (control) and SLC-selected samples were taken for various analyses as described below. The uncentrifuged samples were analyzed first, while the SLC samples were being prepared; approximately 2 to $3 \mathrm{~h}$ elapsed between analyzing the 2 sets of samples.

\section{Sperm Concentration}

The concentration of spermatozoa in both the unselected and SLC-selected samples was measured using a Nucleocounter-SP 100 cell counter (Chemometec,
Allerød, Denmark) according to the manufacturer's instructions. An aliquot $(50 \mu \mathrm{L})$ from each sample was mixed with $5 \mathrm{~mL}$ of reagent S100 in a sample cup. A cassette containing propidium iodide (PI) was filled with the mixture before being inserted into the Nucleocounter-SP100. After $\sim 30 \mathrm{~s}$, the total cell count was displayed.

\section{Sperm Morphology}

Briefly, aliquots of the fresh, extended ejaculates and SLC-selected sperm preparations were used to prepare air-dried slides for assessment of sperm head shape of 500 spermatozoa at $1,000 \times$ magnification, following staining. In addition, aliquots were fixed in buffered formaldehyde solution for counting of 200 spermatozoa on wet smears $(1,000 \times)$. The evaluation was carried according to Morrell et al. (2009b). The mean proportion of morphologically normal spermatozoa was estimated as the remaining proportion left from total abnormal spermatozoa, including those spermatozoa with distal cytoplasmic droplets, which were considered to be normal. The morphological examinations were performed by skilled staff from the sperm laboratory at the Swedish University of Agricultural Sciences, which acts as a reference laboratory for Sweden.

\section{Assessment of Plasma Membrane Integrity}

Assessment of plasma membrane integrity by flow cytometer using SYBR14 and PI staining was performed according to the procedure described by Johannisson et al. (2009), with slight adaptations for samples from bulls. Briefly, aliquots from the unselected and SLCselected samples were extended with buffer B (patent pending; J. M. Morrell and H. Rodriguez-Martinez) to a concentration of approximately $2 \times 10^{6}$ sperm cells/ $\mathrm{mL}$. A $300-\mu \mathrm{L}$ aliquot of each sample was stained with $0.6 \mu \mathrm{L}$ of $0.02 \mu M$ SYBR14 and $3 \mu \mathrm{L}$ of $12 \mu M$ PI (Live-Dead Sperm Viability Kit L-7011; Invitrogen, Eugene, OR) and incubated at $38^{\circ} \mathrm{C}$ for $10 \mathrm{~min}$. The stained samples were evaluated using a BD LSR flow cytometer (Becton Dickinson, San Jose, CA). Excitation was induced by an argon-ion laser (488 nm). Green fluorescence was detected with a fluorescence channel (FL1) band-pass filter $(530 / 30 \mathrm{~nm})$ and red fluorescence was measured using a FL3 long-pass filter $(>670 \mathrm{~nm})$. A total of 50,000 sperm-specific events was evaluated and calculated as percentages of living, dead, and dying cells. The cells were classified, according to the degree of intactness of the plasma membrane, as living (SYBR14-positive/PI-negative), dead (SYBR14negative/PI-positive), and dying (SYBR14-positive/ PI-positive). 
CASA

Computer-assisted sperm analysis was performed using a SpermVision analyzer (Minitüb $\mathrm{GmbH}$ ), connected to an Olympus BX 51 microscope (Olympus, Tokyo, Japan) with a heated stage $\left(38^{\circ} \mathrm{C}\right)$. Aliquots $(6$ $\mu \mathrm{L}$ ) of the sperm samples were pipetted on to a warm glass slide and an 18- $\times 18$-mm coverslip was placed on top. Sperm motility was analyzed using the SpermVision software program for bull spermatozoa. For the purposes of this experiment, only total and progressive motility are reported.

\section{Sperm Chromatin Structure Assay}

Sperm chromatin integrity was evaluated according to the sperm chromatin structure assay (SCSA) method of Evenson et al. (2002) with slight modifications. Briefly, aliquots from 60 uncentrifuged sperm samples and their corresponding SLC-selected sperm samples were mixed 1:1 with Tris-sodium chloride-EDTA (TNE) buffer $(0.15 \mathrm{~mol} / \mathrm{L} \mathrm{NaCl}, 0.01 \mathrm{~mol} / \mathrm{L}$ Tris-HCl, $1 \mathrm{mmol} / \mathrm{L}$ EDTA, pH 7.4). An aliquot $(0.2 \mathrm{~mL})$ of each sample was snap-frozen in liquid nitrogen and stored at $-80^{\circ} \mathrm{C}$ until required for subsequent sperm chromatin structure assay. Before staining, the samples were thawed on crushed ice. The thawed spermatozoa $(10 \mu \mathrm{L})$, extended with TNE buffer $(90 \mu \mathrm{L})$, were subjected to partial DNA denaturation in situ (by mixing with $0.2 \mathrm{~mL}$ of a low $\mathrm{pH}$ detergent solution containing $0.17 \%$ Triton $\mathrm{X}-100,0.15 \mathrm{~mol} / \mathrm{L} \mathrm{NaCl}$, and $0.08 \mathrm{~mol} / \mathrm{L} \mathrm{HCl} ; \mathrm{pH} 1.2$ ), followed $30 \mathrm{~s}$ later by staining with $0.6 \mathrm{~mL}$ of acridine orange $(6 \mu \mathrm{g} / \mathrm{mL}$ in $0.1 \mathrm{~mol} / \mathrm{L}$ citric acid, $0.2 \mathrm{~mol} / \mathrm{L}$ $\mathrm{Na}_{2} \mathrm{HPO}_{4}, 1 \mathrm{mmol} / \mathrm{L}$ EDTA, $0.15 \mathrm{~mol} / \mathrm{L} \mathrm{NaCl} ; \mathrm{pH}$ 6.0). The stained samples were analyzed within 3 to $5 \mathrm{~min}$ of acridine orange staining using a FACStar Plus flow cytometer (Becton Dickinson) equipped with standard optics. Acridine orange was excited with an argon ion laser (Innova 90; Coherent, Santa Clara, CA) at 488 $\mathrm{nm}$, running at $200 \mathrm{~mW}$. The fluorescence stability of the flow cytometer was checked daily using standard beads (Fluoresbrite plain YG $1.0 \mu \mathrm{mol} / \mathrm{L}$; Polysciences, Warrington, PA). Equivalent instrument settings were used for all samples. From each sample, a total of 10,000 events were measured at a flow rate of approximately 200 cells/s. Data collection was carried out using CellQuest (version 3.3, Becton Dickinson). Further calculations of SCSA parameters such as DNA fragmentation index [\%DFI; calculated as ratio of the percentage of cells with denatured, single-stranded DNA to total cells acquired (both with stable, double-stranded DNA, and denatured single-stranded DNA)], mean DFI, and SD of DFI were evaluated using FCS Express version 2 (De Novo Software, Thornhill, ON, Canada).

\section{Detection of ROS}

Aliquots from the unselected and SLC-selected samples were extended to a concentration of approximately $2 \times 10^{6}$ sperm cells $/ \mathrm{mL}$ using buffer B and were stained as follows: 2 aliquots $(300 \mu \mathrm{L})$ of extended samples were stained with $9 \mu \mathrm{L}$ of $40 \mu M$ Hoechst 33258 (HO; Sigma, Stockholm, Sweden), $9 \mu \mathrm{L}$ of $40 \mu M$ hydroethidine (HE; Invitrogen), and $9 \mu \mathrm{L}$ of $2 \mathrm{mM} 2^{\prime}, 7^{\prime}$-dichlorodihydrofluorescein diacetate (DCFDA; Invitrogen). In addition to the above stains, $3 \mu \mathrm{L}$ of $20 \mathrm{mM}$ menadione was added to one of the aliquots to stimulate ROS production. The HE and DCFDA stains were used to detect superoxide anion and hydrogen peroxide $\left(\mathrm{H}_{2} \mathrm{O}_{2}\right)$, respectively, whereas $\mathrm{HO}$ was added to permit the simultaneous differentiation of living and dead cells (Guthrie and Welch, 2006). The samples were gently mixed and incubated at $38^{\circ} \mathrm{C}$ for 30 min before being analyzed with a BD LSR flow cytometer (Becton Dickinson). Excitation was with an argon-ion laser (488 nm) and a HeCd laser $(325 \mathrm{~nm})$. Detection of green fluorescence was with a FL1 band-pass filter $(530 / 30 \mathrm{~nm})$, red fluorescence was measured using a FL3 long-pass filter $(>670 \mathrm{~nm})$, and blue fluorescence was detected with a FL4 band-pass filter (510/20 nm). In total, 50,000 sperm specific events were evaluated and calculated as percentages. The spermatozoa detected were classified as live, superoxide-negative; live, superoxide-positive; dead, superoxide-positive; live, $\mathrm{H}_{2} \mathrm{O}_{2}$-negative; live, $\mathrm{H}_{2} \mathrm{O}_{2}$-positive; dead, $\mathrm{H}_{2} \mathrm{O}_{2}$-negative; and dead, $\mathrm{H}_{2} \mathrm{O}_{2-}$ positive by region statistics. Percentages of the total population of sperm-specific events in these regions were calculated.

\section{Assessment of Mitochondrial Membrane Potential}

Mitochondrial membrane potential (MMP) of sperm cells was measured by using the lipophilic cationic probe JC-1, according to the method described by Cossarizza et al., (1993). Briefly, aliquots of unselected and SLCselected samples were diluted with buffer B to obtain a concentration of approximately $2.5 \times 10^{6}$ sperm cells/ $\mathrm{mL}$, and an aliquot $(300 \mu \mathrm{L})$ of the diluted sample was stained with $1.2 \mu \mathrm{L}$ of $3 \mathrm{mM} \mathrm{JC}-1$ and incubated at $38^{\circ} \mathrm{C}$ for $40 \mathrm{~min}$. The stained samples were analyzed with a BD LSR flow cytometer (Beckon Dickinson). Excitation of stained cells was obtained by the instrument's argon-ion laser (488 nm). Emitted fluorescence was detected using both FL1 $(530 / 30 \mathrm{~nm})$ and FL2 $(585 \mathrm{~nm})$ filters. Green emission was analyzed in FL1 and greenish-orange in FL2. A total of 30,000 cells were evaluated and classified as percentages (CellQuest, version 3.3; Beckon Dickinson) of 2 distinct groups: sperm cells with high respiratory activity (orange fluores- 
cence) and those with low respiratory activity (green fluorescence).

\section{Estimation of Global Protein Tyrosine Phosphorylation}

Global protein tyrosine phosphorylation in both unselected and SLC-selected bull sperm samples was analyzed using flow cytometry as previously described (Sidhu et al., 2004; Piehler et al., 2006) with modifications. Briefly, aliquots of unselected and SLCselected samples were diluted with buffer B to obtain a concentration of approximately $8 \times 10^{6}$ sperm cells/ $\mathrm{mL}$, and $7 \mu \mathrm{L}$ of PI was added to the sperm suspension, which was then incubated at $37^{\circ} \mathrm{C}$ for $15 \mathrm{~min}$ and centrifuged at $300 \times g$ at $20^{\circ} \mathrm{C}$ for $10 \mathrm{~min}$. After centrifugation, the supernatant was discarded and the spermatozoa pellet was resuspended in $1 \mathrm{~mL}$ of buffer B. Each sample was split into 2 parts $(500 \mu \mathrm{L}$ each $)$ and centrifuged $(300 \times g ; 10 \mathrm{~min})$ and the supernatant was removed. The pellet was resuspended in 500 $\mu \mathrm{L}$ of $1 \%$ paraformaldehyde in PBS and incubated at $4^{\circ} \mathrm{C}$ for $30 \mathrm{~min}$. The suspension was subsequently centrifuged at $1,000 \times g$ for $3 \mathrm{~min}$ and the supernatant was removed. The pellet was resuspended in $1 \mathrm{~mL}$ of $0.05 \%$ saponin (Sigma) in PBS and incubated for 10 min at room temperature to permeabilize the cells. The sample was centrifuged again $(300 \times g ; 10 \mathrm{~min})$, the supernatant discarded, and the pellet suspended in $500 \mu \mathrm{L}$ of PBS containing $0.1 \%$ BSA (Sigma) and $0.1 \%$ Tween 20 to block nonspecific binding sites. Each sample mixture was again incubated at room temperature for $30 \mathrm{~min}$, centrifuged $(300 \times g ; 10 \mathrm{~min})$ and the supernatant was removed. Fluorescein isothiocyanate (FITC)-conjugated monoclonal anti-phosphotyrosine antibody produced in mouse clone PT-66 (Sigma) was added $(100 \mu \mathrm{L}$, diluted1:300 in PBS) to one part of the

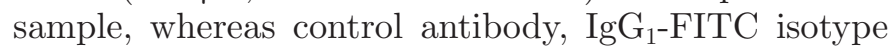
control from murine myeloma, clone MOPC21 (Sigma), was added $(100 \mu \mathrm{L}$, diluted $1: 20$ in PBS) to the other part, and both tubes were incubated at room temperature in the dark for $20 \mathrm{~min}$. Subsequently, $1 \mathrm{~mL}$ of PBS was added to each tube before centrifugation at $300 \times$ $g$ for $10 \mathrm{~min}$. After removing the supernatant, the pellet was resuspended in $500 \mu \mathrm{L}$ of PBS and analyzed by flow cytometry. Data from at least 30,000 events were analyzed per sample. Acquisitions and analyses were made using Cell Quest 3.3 software (Becton Dickinson).

\section{Statistics}

Data analysis was performed using GraphPad Prism (version 5; GraphPad Software Inc., La Jolla, CA). Sperm morphology, plasma membrane integrity (vi- ability), chromatin structure, and motility data were analyzed using 2-way ANOVA. To analyze ROS production, MMP, and protein tyrosine phosphorylation data, $t$-test was performed. Correlation analyses were performed on motility versus viability, ROS (live, superoxide-positive; live, $\mathrm{H}_{2} \mathrm{O}_{2}$-negative) versus motility, ROS versus chromatin structure, MMP versus motility, and MMP versus protein tyrosine phosphorylation assays. In all cases, significance was set at $P \leq 0.05$.

\section{RESULTS}

\section{Sperm Yield and Morphology}

The median yield after SLC was 61\% (range: 30 to $87 \%$ ). The proportions of spermatozoa with normal morphology in unselected and SLC-selected samples are shown in Figure 1. The difference in the mean proportion of morphologically normal spermatozoa between unselected and SLC-selected samples was not significant, although it was increased in the SLC samples compared with uncentrifuged controls of 4 bulls (bull no. $3,6,11$, and 18 )

\section{Sperm Membrane Integrity and Motility}

Mean percentages of viable spermatozoa in unselected and corresponding SLC-selected samples are shown in Table 1. We observed no significant differences between the unselected and SLC-selected samples. A significant difference $(P<0.0001)$ was observed between bulls.

Table 1 shows the results of the total and progressive motility assessment for unselected and SLC-selected sperm samples. Neither total nor progressive sperm motility was significantly different in the unselected and SLC-selected spermatozoa. Furthermore, no significant difference was observed between bulls.

\section{Chromatin Structure}

A significantly lower \%DFI $(P=0.001)$ was observed in SLC-selected spermatozoa compared with unselected spermatozoa, and significant differences $(P<0.0001)$ were seen between bulls (Figure 2).

\section{ROS Production}

Figure $3 \mathrm{~A}$ and $3 \mathrm{~B}$ show the proportion of (mean \pm SD) of ROS-containing spermatozoa with and without menadione. Viability measured with Hoechst 33258 (HO) in the ROS assay was compared with the overall means for living spermatozoa from the SYBR14-PI staining. A lower percentage of living spermatozoa was observed in both unselected and SLC-selected 


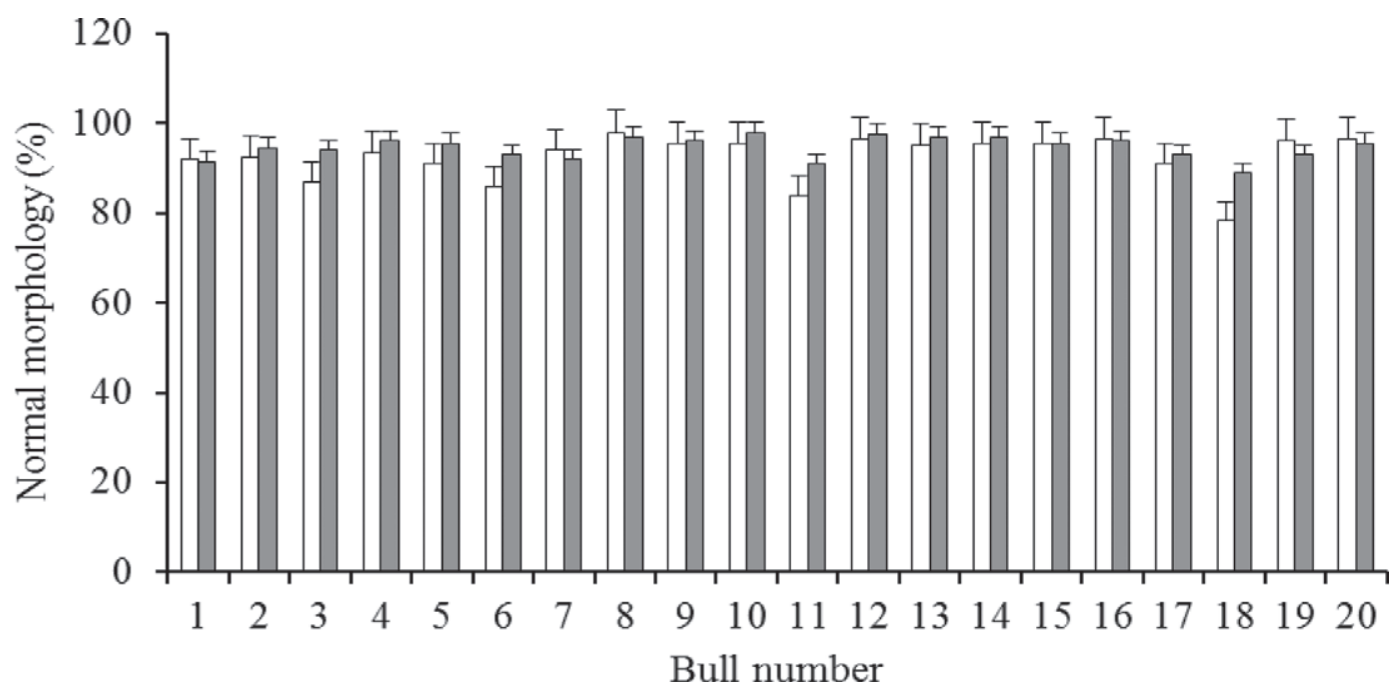

Figure 1. Sperm morphology in unselected (open bars) and single-layer centrifugation (SLC)-selected (gray bars) samples. Data were analyzed using 2-way ANOVA and comparison of means was done by Bonferroni post hoc test. Values are mean $\pm \mathrm{SD} ; \mathrm{n}=20$ in triplicate. $P=$ 0.10: unselected vs. SLC-selected; $P=0.07$ : bull vs. bull.

samples by Hoechst staining method compared with SYBR14-PI assay. Significantly greater proportions of living superoxide-negative and living $\mathrm{H}_{2} \mathrm{O}_{2}$-negative spermatozoa were found in unselected sperm samples compared with SLC-selected samples, with or without menadione induction of ROS. In both the presence and absence of menadione, a significant increase in the percentage of live superoxide-positive, live $\mathrm{H}_{2} \mathrm{O}_{2}$-positive, dead superoxide-positive, dead $\mathrm{H}_{2} \mathrm{O}_{2}$-positive, and dead $\mathrm{H}_{2} \mathrm{O}_{2}$-negative spermatozoa were found in SLC-selected compared with unselected samples.

\section{MMP and Protein Tyrosine Phosphorylation}

The percentage of spermatozoa with high MMP in unselected samples was $56 \%$, whereas the proportion

Table 1. Effect of single-layer centrifugation (SLC) on sperm cell viability and motility (total and progressive) ${ }^{1}$

\begin{tabular}{|c|c|c|c|c|c|c|}
\hline \multirow[b]{2}{*}{ Bull No. } & \multicolumn{2}{|c|}{ Viability $^{2}(\%)$} & \multicolumn{2}{|c|}{ Total motility ${ }^{3}(\%)$} & \multicolumn{2}{|c|}{ Progressive motility $^{4}(\%)$} \\
\hline & Unselected & SLC-selected & Unselected & SLC-selected & Unselected & SLC-selected \\
\hline 1 & $89.43 \pm 3.24$ & $92.50 \pm 1.69$ & $84.33 \pm 4.93$ & $88 \pm 2.65$ & $82.33 \pm 5.13$ & $82.67 \pm 4.04$ \\
\hline 2 & $89.37 \pm 2.16$ & $92.95 \pm 1.15$ & $84.33 \pm 12.0$ & $80.67 \pm 9.07$ & $82.67 \pm 11.02$ & $78.33 \pm 8.74$ \\
\hline 3 & $85.08 \pm 3.42$ & $91.25 \pm 1.74$ & $84 \pm 3.61$ & $90.67 \pm 3.21$ & $81 \pm 3.0$ & $85.67 \pm 4.93$ \\
\hline 4 & $89.31 \pm 1.52$ & $91.77 \pm 2.26$ & $90 \pm 1.0$ & $90.33 \pm 2.08$ & $88 \pm 1.0$ & $87.33 \pm 2.08$ \\
\hline 5 & $86.44 \pm 4.53$ & $82.88 \pm 5.81$ & $87.33 \pm 1.15$ & $86.33 \pm 1.53$ & $85.67 \pm 1.53$ & $82.67 \pm 2.08$ \\
\hline 6 & $85.31 \pm 3.11$ & $88.32 \pm 1.78$ & $89.67 \pm 2.89$ & $88.67 \pm 3.79$ & $86.67 \pm 2.89$ & $85.33 \pm 2.31$ \\
\hline 7 & $93 \pm 1.59$ & $89.60 \pm 5.32$ & $89 \pm 3.46$ & $88.67 \pm 3.51$ & $86 \pm 2.65$ & $86.33 \pm 10.50$ \\
\hline 8 & $88.38 \pm 8.85$ & $87.47 \pm 6.15$ & $83 \pm 17.35$ & $84 \pm 17.35$ & $81.67 \pm 17.93$ & $78.67 \pm 19.66$ \\
\hline 9 & $90.37 \pm 4.92$ & $86.81 \pm 11.0$ & $85.67 \pm 7.77$ & $87 \pm 11.36$ & $82.67 \pm 11.15$ & $84.33 \pm 11.59$ \\
\hline 10 & $91.71 \pm 2.74$ & $93.41 \pm 1.72$ & $87.33 \pm 6.03$ & $90 \pm 2.65$ & $84.67 \pm 6.51$ & $86.33 \pm 3.21$ \\
\hline 11 & $87.52 \pm 2.17$ & $84.12 \pm 6.11$ & $81.33 \pm 10.02$ & $83.33 \pm 5.69$ & $78.33 \pm 9.61$ & $78 \pm 6.56$ \\
\hline 12 & $83.02 \pm 9.06$ & $85.90 \pm 4.40$ & $84.33 \pm 8.62$ & $81.67 \pm 8.74$ & $81 \pm 8.89$ & $75 \pm 11.27$ \\
\hline 13 & $92.82 \pm 0.73$ & $94.06 \pm 0.85$ & $90 \pm 2.65$ & $91 \pm 2.65$ & $87.33 \pm 2.89$ & $86.67 \pm 2.52$ \\
\hline 14 & $87.27 \pm 2.95$ & $89.84 \pm 3.15$ & $82.33 \pm 4.04$ & $89.67 \pm 0.58$ & $79 \pm 3.61$ & $85 \pm 2.0$ \\
\hline 15 & $92.09 \pm 3.65$ & $93.01 \pm 4.16$ & $88.33 \pm 3.51$ & $89.33 \pm 4.04$ & $86 \pm 3.61$ & $85.67 \pm 4.51$ \\
\hline 16 & $93.81 \pm 2.30$ & $95.18 \pm 0.16$ & $90 \pm 3.61$ & $93 \pm 1.73$ & $88.33 \pm 3.51$ & $89.67 \pm 1.53$ \\
\hline 17 & $85.09 \pm 5.37$ & $79.44 \pm 9.31$ & $83 \pm 4.58$ & $83 \pm 2.65$ & $79.67 \pm 5.03$ & $77 \pm 6.0$ \\
\hline 18 & $80.27 \pm 5.25$ & $81.26 \pm 11.1$ & $83 \pm 7.0$ & $88 \pm 2.65$ & $80 \pm 6.56$ & $84.67 \pm 2.52$ \\
\hline 19 & $87.25 \pm 2.73$ & $85.57 \pm 4.40$ & $83.33 \pm 2.08$ & $87.67 \pm 3.79$ & $80 \pm 1.73$ & $83.67 \pm 5.03$ \\
\hline 20 & $92.01 \pm 1.11$ & $88.80 \pm 4.70$ & $91.07 \pm 2.57$ & $91.67 \pm 6.66$ & $89 \pm 3.0$ & $88.33 \pm 7.37$ \\
\hline
\end{tabular}

${ }^{1}$ Values are mean \pm SD. Data were analyzed using 2-way ANOVA and comparison of means was done by Bonferroni post hoc test.

${ }^{2} P=0.91$ : unselected vs. SLC-selected; and $P \leq 0.0001$ : bull vs. bull.

${ }^{3} P=0.19$ : unselected vs. SLC-selected; and $P=0.23$ : bull vs. bull.

${ }^{4} P=0.92$ : unselected vs. SLC-selected; and $P=0.71$ : bull vs. bull. 


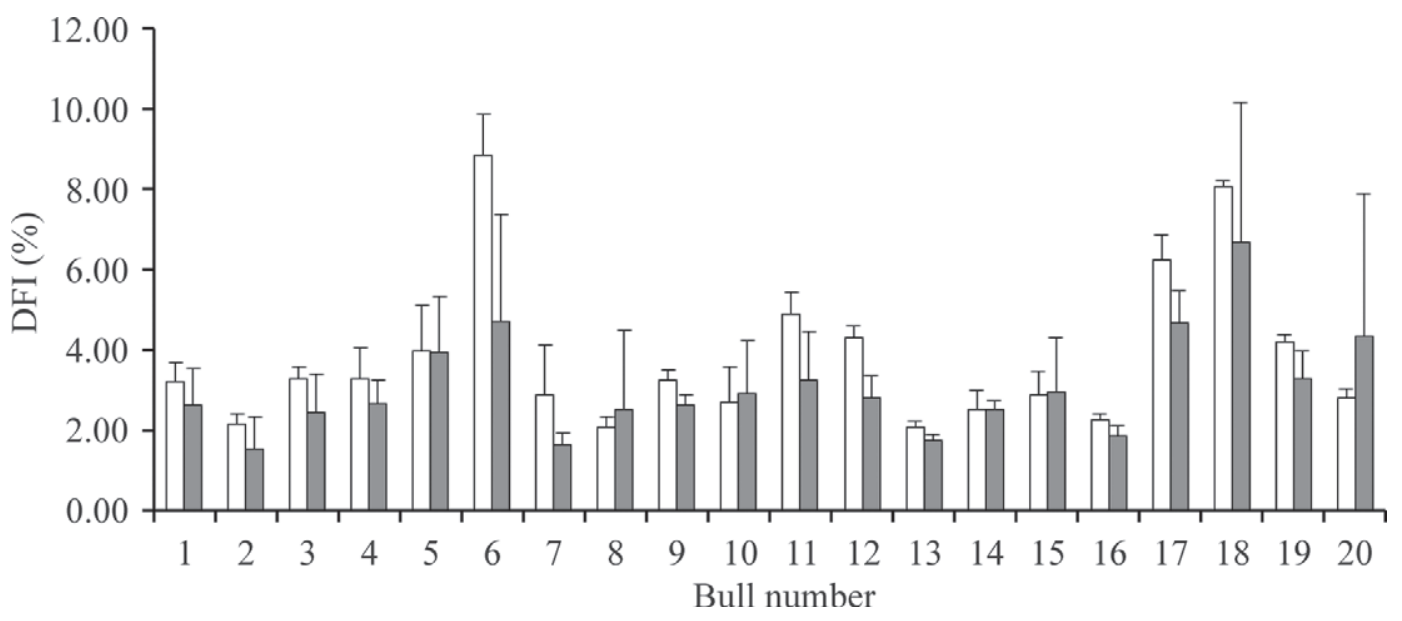

Figure 2. Sperm chromatin integrity in unselected (open bars) and single-layer centrifugation (SLC)-selected (gray bars) samples. Data were analyzed using 2-way ANOVA and comparison of means was done by Bonferroni post hoc test. Values are mean $\pm \mathrm{SD} ; \mathrm{n}=20$ in triplicate. $P$ $<0.001$ : unselected vs. SLC-selected; $P<0.0001$ : bull vs. bull. \%DFI $=$ DNA fragmentation index.

reached $83 \%$ in SLC-selected spermatozoa (Figure 4; $P<0.001)$. The percentage of spermatozoa with low MMP was significantly $(P<0.0001)$ higher in unselected spermatozoa compared with SLC-selected spermatozoa.

The mean proportion of spermatozoa with protein tyrosine phosphorylation was significantly higher in SLC-selected samples compared with unselected samples $(3.75 \pm 0.7 \%$ and $5.81 \pm 1.4 \%$ for unselected and SLC-selected spermatozoa respectively; $P=0.003$ ).

\section{Correlations}

We detected a significant negative correlation between live, superoxide-positive spermatozoa and \%DFI for the SLC-selected spermatozoa $(\mathrm{r}=-0.51, P=0.02)$ but not for unselected samples. A significant positive correlation was also observed between live, $\mathrm{H}_{2} \mathrm{O}_{2}$-negative spermatozoa and total motility for SLC-selected samples $(\mathrm{r}=0.51, P=0.02)$ but not for unselected samples. The correlation between live, $\mathrm{H}_{2} \mathrm{O}_{2}$-negative spermatozoa and progressive motility for SLC-selected samples was also significant $(\mathrm{r}=0.51, P=0.02)$. We found no significant correlations between other variables for either unselected or SLC-selected spermatozoa.

\section{DISCUSSION}

The aims of the present study were to evaluate the changes in bull sperm quality after SLC with the species-specific colloid Androcoll-B. The parameters of sperm quality measured included sperm morphology, viability, motility, chromatin structure, ROS production, MMP, and protein tyrosine phosphorylation.
Neither sperm viability nor total and progressive motility was significantly different between unselected and SLC-selected samples. These observations are consistent with those of Rodriguez-Martinez et al. (1997), who did not detect any effect of colloid centrifugation on bull sperm motility, and with those of van Wienen et al. (2011), who did not observe any effect of SLC on boar sperm viability or total and progressive motility, although there was an increase in linear motility in that study. However, the present results are in contrast to results in stallion and boar spermatozoa where viability and total and progressive motility were improved by SLC selection (Johannisson et al., 2009; Morrell et al., 2009c). Thus, a species difference may be present, possibly due to the better sperm quality in the uncentrifuged semen samples of bulls that have been selected for sperm quality over many generations compared with stallions that are not selected for sperm quality. However, even if the majority of samples did not show an improvement in normal morphology, viability, or motility after SLC, these parameters in the SLC-selected samples from 4 bulls (bull no. 3, 6, 11, and 18) were mostly improved. The \%DFI after SLC in these 4 bulls was lower than in the unselected samples, and high proportions of spermatozoa with high mitochondrial membrane potential were observed in these 4 bulls after SLC.

The SCSA is a potent diagnostic tool to determine the proportion of DNA damage in the spermatozoa of many species (Cordelli et al., 2005), although, in a recent study (Nagy et al., 2013), no differences between subfertile bulls and fertile sires were found for SCSA parameters. In the present study, less DNA damage was observed in SLC-selected spermatozoa than in the 
(A)

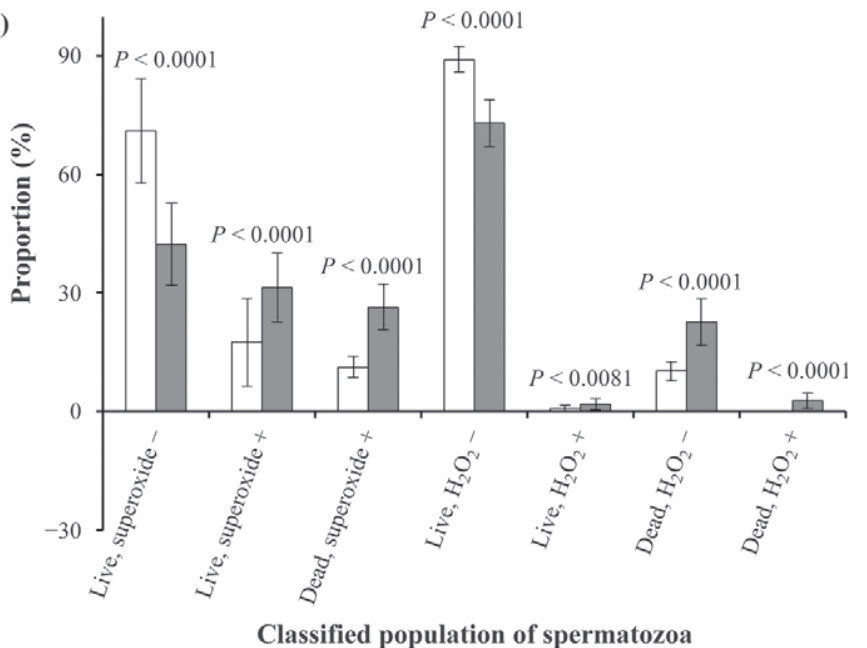

(B)

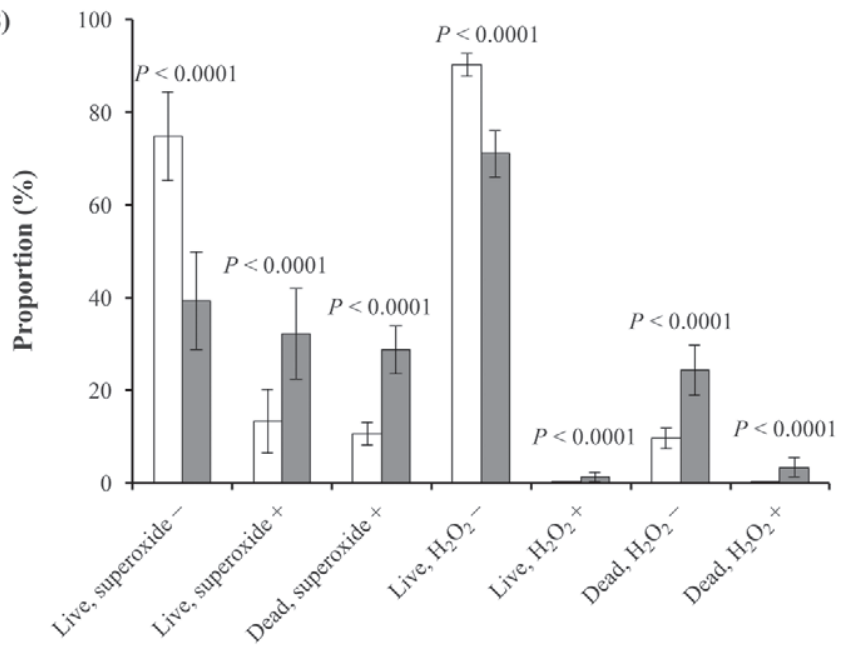

Classified population of spermatozoa

Figure 3. Production of reactive oxygen species (ROS) in unselected (open bars) and single-layer centrifugation (SLC)-selected (gray bars) spermatozoa without (A) and with (B) menadione induction of ROS production. Values are mean $\pm \mathrm{SD}$. Student's $t$-test was used for comparison. $P$-value indicates significant difference between unselected and SLC-selected samples; $\mathrm{n}=20$ in triplicate.

unselected samples. One explanation for this result could be that SLC selects mature spermatozoa with intact chromatin, removing them from immature and damaged spermatozoa and from the seminal plasma environment (Colleu et al., 1996). These results are consistent with previous results with stallion semen (Johannisson et al., 2009; Morrell et al., 2009b, 2011).

Regarding ROS production, fewer ROS were observed in unselected samples compared with SLCselected samples. The SLC-selected samples apparently had a higher proportion of dead spermatozoa containing superoxide and $\mathrm{H}_{2} \mathrm{O}_{2}$ radicals than the unselected samples. However, different proportions of living spermatozoa were detected for the ROS staining method

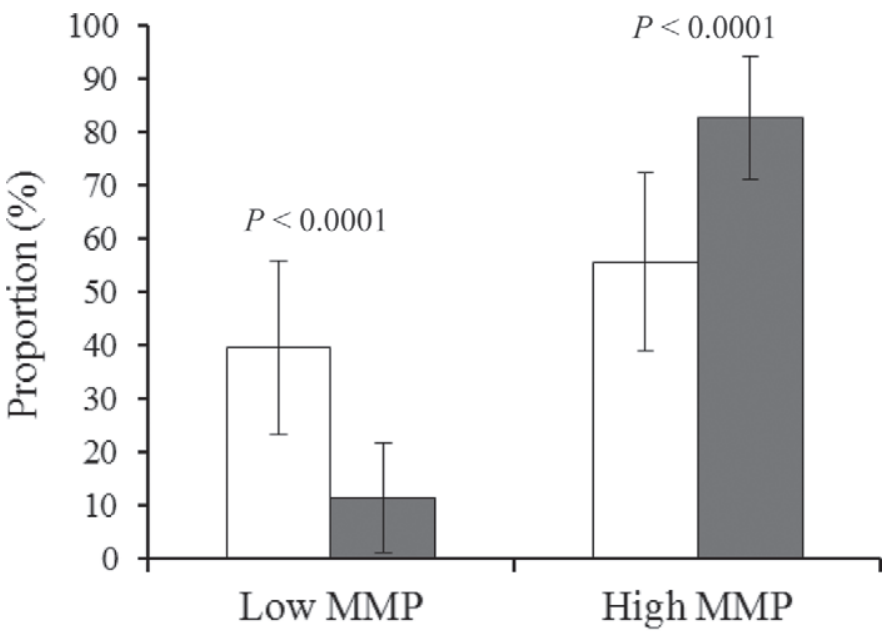

Classified population of spermatozoa

Figure 4. Mitochondrial membrane potential (MMP) in unselected (open bars) and single-layer centrifugation (SLC)-selected (gray bars) spermatozoa. Values are mean $\pm \mathrm{SD}$. Student's $t$-test was used for comparison. $P$-value indicates significant difference between unselected and SLC-selected samples; $\mathrm{n}=20$ in triplicate.

and the SYBR14-PI staining method, despite the analyses being performed on the same samples and on the same day, indicating a discrepancy between staining methods. Because the different incubation times for the 2 techniques (10 min for SYBR-PI and $30 \mathrm{~min}$ for HO-HE-DCFDA) might have contributed to the difference in viability results, we performed additional experiments in which both staining protocols were used with the same incubation time (i.e., first for $10 \mathrm{~min}$ and then for $30 \mathrm{~min}$ ) and found that incubation time did not affect the viability of the spermatozoa (data not presented). This result suggests that HO-HE-DCFDA staining had a negative effect on sperm viability. At physiological levels, free radicals including superoxide and $\mathrm{H}_{2} \mathrm{O}_{2}$ control sperm maturation, capacitation, hyperactivation, acrosome reaction, and sperm-oocyte fusion (de Lamirande et al., 1997). Under normal physiological conditions, a balance exists between oxidants and antioxidants. However, when ROS levels overwhelm the antioxidant defense system, pathological condition results (Kothari et al., 2010). The fact that no loss in motility and chromatin integrity or structure of spermatozoa was observed in this study indicates that the ROS generated did not damage spermatozoa under the experimental conditions reported here. In fact, SLC may select metabolically active spermatozoa that are generating ROS. Our findings are in accord with the reports of van Wienen et al. (2011), who observed no difference in total motility among the unselected and SLC-selected samples and increased proportions of superoxide in SLC-selected samples of boar semen. How- 
ever, the present results differ from those observed with stallion spermatozoa, where decreased proportions of $\mathrm{H}_{2} \mathrm{O}_{2}$-containing spermatozoa were observed after SLC compared with uncentrifuged controls. Whether these differences reflect true species differences or merely differences in the antioxidant characteristics of different semen extenders is not known at present but is under investigation.

Reduced MMP has frequently been associated with sperm abnormalities (Marchetti et al., 2002) and a reduction in fertility (Gallon et al., 2006). On the basis of this evidence, measurement of MMP can be considered a suitable test to determine sperm quality (Barroso et al., 2006). It has been suggested that MMP should be used as a complement to traditional semen analysis in routine examination to evaluate male infertility (Gallon et al., 2006). Results from the current study suggest that preparing the sperm samples by SLC selects spermatozoa with increased MMP. However, a large difference in the proportion of cells with higher MMP was observed between SLC-selected and unselected samples, too large to be solely due to the selection of robust spermatozoa by SLC. On the other hand, SLC separates spermatozoa from the seminal plasma, which contains decapacitation factors such as phospholipase (Sias et al., 2005), thus allowing the spermatozoa to begin to capacitate and possibly increasing their metabolism. Interestingly, the results from protein tyrosine phosphorylation assay in the present study tended to confirm this possibility.

During sperm capacitation, which is crucial for fertilization, protein tyrosine phosphorylation is a major event related to the functionality of spermatozoa. The present study demonstrated that SLC-selected samples possess a higher content of phosphorylated protein than the unselected samples. Our findings are in contrast with Bucci et al. (2013), who did not observe a difference in protein tyrosine phosphorylation between control and SLC boar sperm samples. This could reflect a difference between species or between semen extenders, because the bull sperm samples in the present study were extended with Andromed.

In summary, SLC of bull spermatozoa extended and stored in Andromed resulted in selection of a sperm population that had high MMP, a higher content of phosphorylated protein, and more ROS than control samples. Sperm chromatin damage was lower in the SLC samples, although sperm viability and motility did not differ between SLC samples and controls. These observations suggest that SLC could be a useful procedure to improve sperm quality in some bulls, although further studies are needed.

In the horse, where AI trials have been carried out with SLC-selected sperm samples, SLC has been shown to improve the sperm quality of problem ejaculates. Pregnancy rates are also higher when SLC-selected samples prepared from "normal" ejaculates are used for AI compared with the corresponding untreated controls (fresh and cooled sperm doses). Furthermore, SLC-selected stallion and boar spermatozoa survive freezing better and survive longer after thawing than unselected controls (Morrell, 2011; Martinez-Alborcia et al., 2013). Thus, our previous studies in other species indicate that SLC improves both sperm quality and sperm survival, and that pregnancy rates are higher after AI. The potential benefit of SLC in preparing bull spermatozoa is that a similar improvement in pregnancy rate or, more importantly, an increase in embryo survival after AI, could result from the improvement in sperm quality shown in laboratory analyses. Singlelayer centrifugation-selected bull sperm samples have previously been shown to give good fertilization results in in vitro fertilization (Thys et al., 2009).

Early capacitation could be an advantage in in vitro fertilization but not for AI unless the early-capacitated spermatozoa survived cryopreservation better than untreated spermatozoa. However, early capacitation was not observed for stallion spermatozoa. Thus, early capacitation may only be a feature of SLC using bull sperm extended in the extender Andromed. An AI trial is currently underway to address some of these factors.

\section{ACKNOWLEDGMENTS}

The study was funded by the Swedish Research Council for the Environment, Agricultural Sciences and Spatial Planning (FORMAS), project number 2212010-1241, awarded to J. M. Morrell. We are grateful to Karin Selin-Wretling [Clinical Sciences, Swedish University of Agricultural Sciences (SLU), Uppsala, Sweden] for her skilled morphological analysis, to Celina Abrahams and Denise Laskowski (both at Clinical Sciences, SLU) for laboratory assistance, and to the personnel at Viking Genetics (Falkenberg, Sweden) for their help in sending us the semen samples used in this project.

\section{REFERENCES}

Barroso, G., S. Taylor, M. Morshedi, F. Manzur, F. Gaviño, and S. Oehninger. 2006. Mitochondrial membrane potential integrity and plasma membrane translocation of phosphatidylserine as early apoptotic markers: A comparison of two different sperm subpopulations. Fertil. Steril. 85:149-154.

Bucci, D., M. Spinaci, J. Morrell, C. Vallorani, C. Tamanini, R. Guidetti, and G. Galeati. 2013. Effects of single layer centrifugation with Androcoll-P on boar sperm. Anim. Reprod. Sci. 138:276-281.

Colleu, D., D. Lescoat, and J. Gouranton. 1996. Nuclear maturity of human spermatozoa selected by swim-up or by Percoll gradient centrifugation procedures. Fertil. Steril. 65:160-164.

Cordelli, E., P. Eleuteri, G. Leter, M. Rescia, and M. Spanò. 2005. Flow cytometry applications in the evaluation of sperm quality: 
Semen analysis, sperm function and DNA integrity. Contraception 72:273-279.

Cossarizza, A., M. Baccarani-Contri, G. Kalashnikova, and C. Franceschi. 1993. A new method for the cytofluorimetric analysis of mitochondrial membrane potential using the J-aggregate forming lipophilic cation 5,5',6,6'-tetrachloro-1,1',3,3'-tetraethylbenzimidaz olcarbocyanine iodide (JC-1). Biochem. Biophys. Res. Commun. 197:40-45.

de Lamirande, E., H. Jiang, A. Zini, H. Kodama, and C. Gagnon 1997. Reactive oxygen species and sperm physiology. Rev. Reprod. 2:48-54.

Evenson, D. P., K. L. Larson, and L. K. Jost. 2002. Sperm chromatin structure assay: Its clinical use for detecting sperm DNA fragmentation in male infertility and comparisons with other techniques. J. Androl. 23:25-43.

Gallon, F., C. Marchetti, N. Jouy, and P. Marchetti. 2006. The functionality of mitochondria differentiates human spermatozoa with high and low fertilizing capability. Fertil. Steril. 86:1526-1530.

Guthrie, H. D., and G. R. Welch. 2006. Determination of intracellular reactive oxygen species and high mitochondrial membrane potential in Percoll-treated viable boar sperm using fluorescenceactivated flow cytometry. J. Anim. Sci. 84:2089-2100.

Hallap, T., Ü. Jaakma, and H. Rodriguez-Martinez. 2006. Changes in semen quality in Estonian Holstein AI bulls at 3, 5 and 7 years of age. Reprod. Domest. Anim. 41:214-218.

Hallap, T., S. Nagy, M. Håård, Ü. Jaakma, A. Johannisson, and H. Rodriguez-Martinez. 2005. Sperm chromatin stability in frozenthawed semen is maintained over age in AI bulls. Theriogenology 63:1752-1763.

Hossain, M. S., A. Johannisson, M. Wallgren, S. Nagy, A. P. Siqueira, and H. Rodriguez-Martinez. 2011. Flow cytometry for the assessment of animal sperm integrity and functionality: State of the art. Asian J. Androl. 13:406-419.

Jiménez-Rabadán, P., M. Ramón, O. García-Álvarez, A. MarotoMorales, E. del Olmo, M. D. Pérez-Guzmán, A. Bisbal, M. R. Fernández-Santos, J. J. Garde, and A. J. Soler. 2012. Effect of semen collection method (artificial vagina vs. electroejaculation), extender and centrifugation on post-thaw sperm quality of BlancaCeltibérica buck ejaculates. Anim. Reprod. Sci. 132:88-95.

Johannisson, A., J. M. Morrell, J. Thorén, M. Jönsson, A. M. Dalin, and H. Rodriguez-Martinez. 2009. Colloidal centrifugation with Androcoll-E $\mathrm{E}^{\mathrm{TM}}$ prolongs stallion sperm motility, viability and chromatin integrity. Anim. Reprod. Sci. 116:119-128.

Kothari, S., A. Thompson, A. Agarwal, and S. S. du Plessis. 2010. Free radicals: Their beneficial and detrimental effects on sperm function. Indian J. Exp. Biol. 48:425-435.

Marchetti, C., G. Obert, A. Deffosez, P. Formstecher, and P. Marchetti. 2002. Study of mitochondrial membrane potential, reactive oxygen species, DNA fragmentation and cell viability by flow cytometry in human sperm. Hum. Reprod. 17:1257-1265.

Martinez-Alborcia, M. J., J. M. Morrell, I. Barranco, C. Maside, M. A. Gil, I. Parrilla, J. M. Vazquez, E. A. Martinez, and J. Roca. 2013. Suitability and effectiveness of single layer centrifugation using Androcoll-P in the cryopreservation protocol for boar spermatozoa. Anim. Reprod. Sci. 140:173-179.

Morrell, J. M. 2011. Biomimetics in action: Practical applications of single layer centrifugation for equine breeding. J. Vet. Sci. Technol. 2:107. http://dx.doi.org/10.4172/2157-7579.1000107.

Morrell, J. M., A. M. Dalin, and H. Rodriguez-Martinez. 2009a. Comparison of density gradient and single layer centrifugation of stallion spermatozoa: Yield, motility and survival. Equine Vet. J. $41: 53-58$

Morrell, J. M., B. M. Garcia, F. J. Pena, and A. Johannisson. 2011. Processing stored stallion semen doses by single layer centrifugation. Theriogenology 76:1424-1432.
Morrell, J. M., A. Johannisson, A. M. Dalin, and H. Rodriguez-Martinez. 2009b. Morphology and chromatin integrity of stallion spermatozoa prepared by density gradient and single layer centrifugation through silica colloids. Reprod. Domest. Anim. 44:512-517.

Morrell, J. M., and H. Rodriguez-Martinez. 2009. Biomimetic techniques for improving sperm quality in animal breeding: A review. Open Androl. J. 1:1-91.

Morrell, J. M., and H. Rodriguez-Martinez. 2011. Practical applications of sperm selection techniques as a tool for improving reproductive efficiency. Vet. Med. Int. 2011:894767. http://dx.doi. org $/ 10.4061 / 2011 / 894767$.

Morrell, J. M., F. Saravia, M. van Wienen, M. Wallgren, and H. Rodriguez-Martinez. 2009c. Selection of boar spermatozoa using centrifugation on a glycidoxypropyltrimethoxysilane-coated silica colloid. J. Reprod. Dev. 55:547-552.

Morrell, J. M., G. Stuhtmann, S. Meurling, A. Lundgren, C. Winblad, B. Macias Garcia, and A. Johannisson. 2014. Sperm yield after single layer centrifugation with Androcoll-E is related to the potential fertility of the original ejaculate. Theriogenology http:// dx.doi.org/10.1016/j.theriogenology.2014.01.013.

Nagy, S., A. Johannisson, T. Wahlsten, R. Ijas, M. Andersson, and H. Rodriguez-Martinez. 2013. Sperm chromatin structure and sperm morphology: Their association with fertility in AI-dairy Ayrshire sires. Theriogenology 79:1153-1161.

Piehler, E., A. M. Petrunkina, M. Ekhlasi-Hundrieser, and E. TöpferPetersen. 2006. Dynamic quantification of the tyrosine phosphorylation of the sperm surface proteins during capacitation. Cytometry A 69:1062-1070.

Rodriguez-Martinez, H., B. Larsson, B. R. Zhang, and L. Söderquist. 1997. In vitro assessment of viability and fertilizing capacity of bull spermatozoa. J. Reprod. Dev. 43:1-11.

Sias, B., F. Ferrato, M.-T. Pellicer-Rubio, Y. Forgerit, P. Guillouet, B. Leboeuf, and F. Carrière. 2005. Cloning and seasonal secretion of the pancreatic lipase-related protein 2 present in goat seminal plasma. Biochim. Biophys. Acta 1686:169-180.

Sidhu, K. S., K. E. Mate, T. Gunasekera, D. Veal, L. Hetherington, M. A. Baker, R. J. Aitken, and J. C. Rodger. 2004. A flow cytometric assay for global estimation of tyrosine phosphorylation associated with capacitation of spermatozoa from two marsupial species, the tammar wallaby (Macropus eugenii) and the brushtail possum (Trichosurus vulpecula). Reproduction 127:95-103.

Söderquist, L., L. Janson, M. Håård, and S. Einarsson. 1996. Influence of season, age, breed and some other factors on the variation in sperm morphological abnormalities in Swedish dairy AI bulls. Anim. Reprod. Sci. 44:91-98.

Söderquist, L., H. Rodriguez-Martinez, M. G. H. Håård, and N. Lundeheim. 1997. Seasonal variation in sperm morphology in proven Swedish dairy AI bulls. Reprod. Domest. Anim. 32:263-265.

Thys, M., L. Vandaele, J. M. Morrell, J. Mestach, A. van Soom, M. Hoogewijs, and H. Rodriguez-Martinez. 2009. In vitro fertilising capacity of frozen-thawed bull spermatozoa selected by single-layer glycidoxypropyltrimethoxysilane-coated silica colloidal centrifugation. Reprod. Domest. Anim. 44:390-394.

van Wienen, M., M. Wallgren, and J. M. Morrell. 2011. Single layer centrifugation with Androcoll-P can be scaled-up to process larger volumes of boar semen. ISRN Vet. Sci. 2011:548385. http:// dx.doi.org/10.5402/2011/548385.

Verstegen, J., M. Iguer-Ouada, and K. Onclin. 2002. Computer assisted semen analyzers in andrology research and veterinary practice. Theriogenology 57:149-179. 